\title{
Descriptive Design Structure Matrices for Modelling Infrastructure Interdependencies in Community Recovery
}

\author{
Rameez R. Qureshi ${ }^{1}$, David N. Ford ${ }^{1}$, Charles M. Wolf ${ }^{1}$ \\ ${ }^{1}$ Texas A\&M University
}

\begin{abstract}
Interdependencies between infrastructure sectors impact the recovery of communities in the post-disaster period. These interdependencies give rise to multiple feedback loops that drive and constrain recovery. Community leaders tasked with developing a recovery plan must allocate limited resources over time among numerous interacting sectors that need assistance. However, the interdependencies make it difficult to size and sequence the needs. A Descriptive Design Structure Matrix (DDSM) was developed to understand the impacts of community interactions on recovery and resourcing. The DDSM's simple, logical descriptions of infrastructure interactions help identify recovery bottlenecks. Community interactions were analyzed using Cambridge Advanced Modeler. Governance, Electric Power, Commerce, Road Transportation, and Workforce Population were identified as the five most critical sectors that interact with each other to form a core set of causal feedback loops for recovery. The DDSM model can complement existing resource allocation methodologies by providing a systematic and structured approach to sequence resources for a quick recovery from disasters.
\end{abstract}

Keywords: Interdependencies, Community Recovery, Resilience, DSM, Disasters

\section{Introduction}

Disaster experiences, as described by various international agencies, are broadly categorized into Preparation, Mitigation, Disaster, Response, and Recovery phases (National Governors' Association (NGA) 1979). The current work focusses on the community recovery phase. Recovery efforts seek to restore the livelihoods of disaster victims by returning the community to conditions equal to or better than those before the disaster (Ford and Keith 2016). However, limited resources in the post-disaster period and community interdependencies prevent the simultaneous rebuilding of all the damaged components of a community - an approach that would undoubtedly speed recovery. Local government agencies responsible for recovery must decide what community sectors to prioritize to facilitate a fast and sustainable recovery. The level and scale of damage and pre-existing community conditions also contribute to these challenges. Community sectors, due to their interdependencies, make it particularly difficult to decide on the prioritization and allocation of resources. For example, economic recovery is dependent on the interaction and synergistic effects of multiple efforts, including restoring public infrastructure, rebuilding housing, and reopening educational institutions. These interactions need to be considered to develop effective recovery strategies and can have significant policy implications. 
To illustrate these recovery interactions, Figure 1 uses a causal loop diagram (CLD) to describe some of the community recovery causal links and feedback loops that they create. In combination, these variables, links, and loops represent a feedback-based theory of community recovery in a reinforcing growth loop, adapted from Ford and Keith (2016) to our context. In the model, residences support the population that engages in commercial activities to increase the production, manufacturing, and construction capability of the community, which further increases the available homes to meet the population needs (Figure 1, Loop R1). Adequate public revenue generated through commerce increases the available funding for developing public health and transportation infrastructure (Figure 1, Loops R2 \& R3). Population growth is limited by crowding due to the community having more residents than it can support (Figure 1, Loop B1). These elements and their interactions describe the recovery of a community (in a highly simplified form) after a disaster. As can be seen from Figure 1, even when there is adequate housing, the lack of public health infrastructure will constrain the population recovery. Similarly, the lack of sufficient transportation in the community will limit the construction of residences due to the unavailability of raw material supplies required for construction.

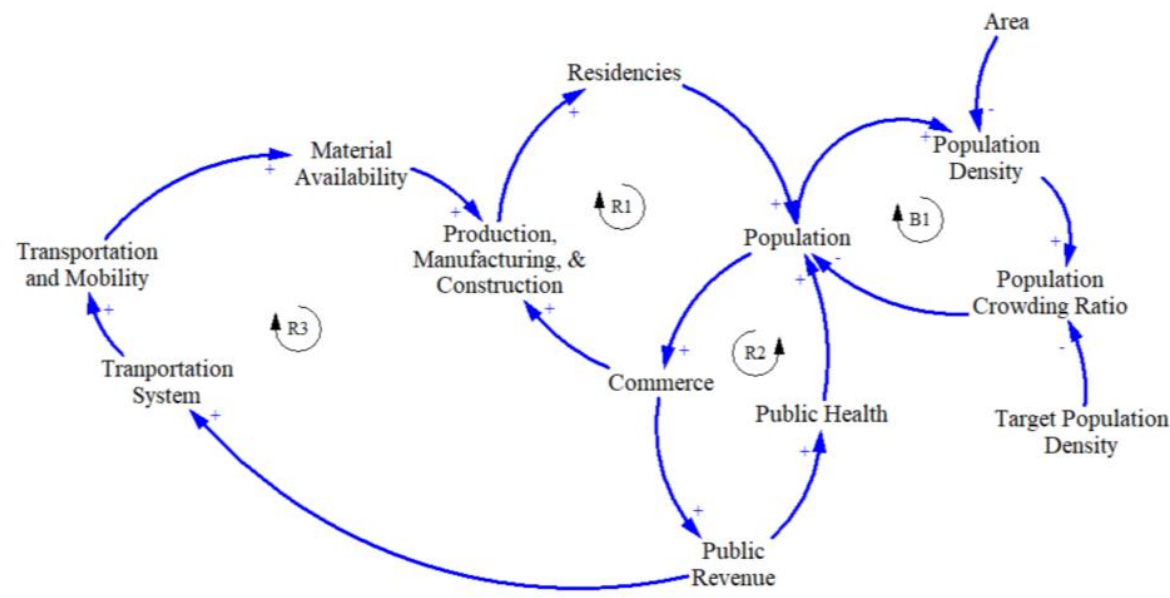

Figure 13 Example Causal Loop Diagram of Interactions in Community Recovery (based on Ford and Keith, 2016) Figure 1 shows just one example of some of the interdependencies between infrastructure systems. Actual communities have many more interdependent components. Some of the feedback loops have few causal links such that the condition of one infrastructure (e.g., demand created by commerce for the availability of road

transportation in the community) directly influences the condition of the other infrastructure (e.g., road transportation capacity to support commercial needs). Other feedback loops have many causal links such that the state of one infrastructure influences the state of other dependent infrastructures in many direct and indirect ways. The interactions create dynamic behaviors and shifts in influence as a community evolves in recovery through rebuilding into quasi-steady-state operations. Therefore, the resource allocation problem in practice is very complicated. Recovery feedback loops can be reinforcing, pushing the system farther and farther in a given direction, or balancing, 
which control behavior. Ford and Keith (2016) and others have modeled recovery as interacting collections of reinforcing growth loops constrained by bottlenecks created by their related balancing loops. Without minimizing the role of balancing feedback loops in community recovery, the current work focuses on the growth loops that, if bottlenecks are more efficiently addressed through improved resource allocation, can improve recovery.

Most of the study is limited to mapping the interdependencies between physical infrastructure and their effects on the community despite the significant research conducted to understand the impacts of infrastructure interdependencies (e.g., see, Amin, 2002; Rand and Fleming, 2019; Ouyang, 2014). A comprehensive understanding of how infrastructure systems interact during disaster recovery to drive and/or constrain recovery of a community is needed. However, most of the existing recovery frameworks such as the World Bank Recovery Guide (GFDRR 2015), FEMA Recovery Framework (Lindell et al. 2006), etc. focus exclusively on processes for developing plans and coordinating recovery efforts. No community-wide models of infrastructure interactions during the recovery phase are known for the strategic allocation of resources across many diverse sectors. For example, should the leaders first rebuild the infrastructure sectors (community infrastructure, water, transport, etc.) or focus on the production sectors (employment and livelihoods, agriculture, etc.)? What if multiple community sectors are affected? The current research helps bridge this gap by identifying and defining a set of relevant community recovery infrastructures, describing and analyzing their interdependencies, and investigating their impacts on recovery. The recovery model provides the lead recovery planning agencies with a scientific approach to allocate limited resources and better understand the effects of different resource allocation strategies.

\section{Research Approach and Methodology}

First, a model of community recovery interactions was developed as a hypothesis of the nature of the interactions. The initial model was tested and improved based on a case study of 2014 flooding in the Indian state of Jammu and Kashmir (J\&K) and applied to illustrate potential uses. The initial model uses Design Structure Matrices (DSM) to describe community infrastructure interactions. Cambridge Advanced Modeler was used to analyze the DSM for developing recovery resourcing strategy recommendations.

A DSM is a two-dimensional matrix representation of the structural or functional interrelationships of objects, tasks, or teams (Browning 2016). The rows and columns in a DSM reflect the parts of an organization or process, and the intersecting cells represent possible interactions of column elements and row elements. Interactions in DSMs are typically represented in a binary fashion, which describes if there is an interaction that exists or not, or with numbers that reflect the strength of interactions. However, such representations may not be useful to community leaders that have little facility with purely quantitative models. Critical for the usefulness of a DSM for community leaders in prioritizing and sequencing resources among sectors, the interdependencies must be easily understood by leaders who often have little or no understanding of built infrastructures or technical models. The DSM developed here uses simple, logical descriptions of community infrastructure interactions that create rebuilding bottlenecks and, therefore, constrain the 
recovery of other components. This DSM is referred to herein as a "Descriptive Design Structure Matrix" (DDSM). The DDSM consists of three parallel matrices. The matrices are parallel in the sense that the elements and their column and row orders are the same in each matrix. Therefore, each set of three cells (one in each matrix) with the same location in their DDSM represents the same community interaction. This parallel structure allows each matrix to be used to investigate multiple interactions at a single level of detail (nontechnical, technical, or sources) and each set of three matrix cells to be used to investigate a specific community interaction at multiple levels of detail.

\subsection{Hypothesis DDSM Development and Testing}

A preliminary DDSM was developed using various World Bank case studies and other literature to identify and describe the interactions among multiple community sectors. Areas used for intersectoral prioritization by the World Bank (GFDRR 2015) were used to identify infrastructure sectors initially. The sectors were aggregated at the community level to describe 16 distinct infrastructure systems that represent the major community sectors, in line with the typical breakdown of programmatic recovery as outlined by the World Bank (GFDRR 2015). These sectors were used as rows and columns of the DDSM. To test the validity of reported interactions in the hypothesis, J\&K was chosen as the case study to test and analyze the community infrastructure system interactions. News articles and other scholarly literature published following the 2014 floods in J\&K, that reported the impacts of disruptions on the community infrastructures were used to identify how the lack of one infrastructure affected the operation of the other sectors. The case study infrastructure interactions were modeled as a DDSM. The case study DDSM was then compared to the hypothesis DDSM to assess the model validity and to improve the model.

\section{The Hypothesis: A Community Recovery DDSM}

The hypothesis consists of a set of community sectors that are most relevant to recovery from disasters structured into a set of these complimentary design structure matrices.

\subsection{Community Recovery Sectors}

Community infrastructure sectors and their interactions that most impact the recovery of a community were identified and represented in the form of a DDSM to generate the critical community sectors and infrastructure services for recovery. The sixteen sectors that describe the recovery of a community are: Housing, Population (Resident and Workforce), Road Transportation, Other Modes of Transportation, Power Generation and Distribution, Fuel Supply and Distribution, Water Supply and Wastewater Treatment, Drainage and Sanitation, Public Health, Commerce, Food, Information and Communications Technology, Tourism, Governance, and Education. Each sector was clearly defined, with notes as needed, based on existing community sector descriptions and modeling needs. Each sector has distinct characteristics and is mutually exclusive to other sectors.

\subsection{The Community Recovery DDSM}

The hypothesis DDSM consists of the sector definitions and three parallel 2-dimensional matrices. The infrastructure sectors were used as the rows and columns for the DDSM. 
Level 1 of the DDSM describes the interactions in text that can be easily understood by community leaders without technical expertise, e.g., the mayor of the community. Level 2 provides detailed explanations of interdependencies that elaborate on the interactions in the Level 1 matrix for technical community leaders (e.g., department heads). The Level 3 matrix provides literature support for each of the reported infrastructure interactions in Levels 1 and Level 2. Each matrix in the DDSM is 16 by 16 and unreadable if shown in its entirety here. Therefore, only example portions are shown. The complete DDSM is available from the authors.

Figure 2 illustrates the interactions with a portion of the Level 1 matrix of the hypothesis DDSM. Interdependencies between these sectors were identified based on the literature and modeler's understanding of the requirements for goods and services for the physical reconstruction and operation of the infrastructure system. An example of the latter is the need for a workforce by the governance infrastructure sectors.

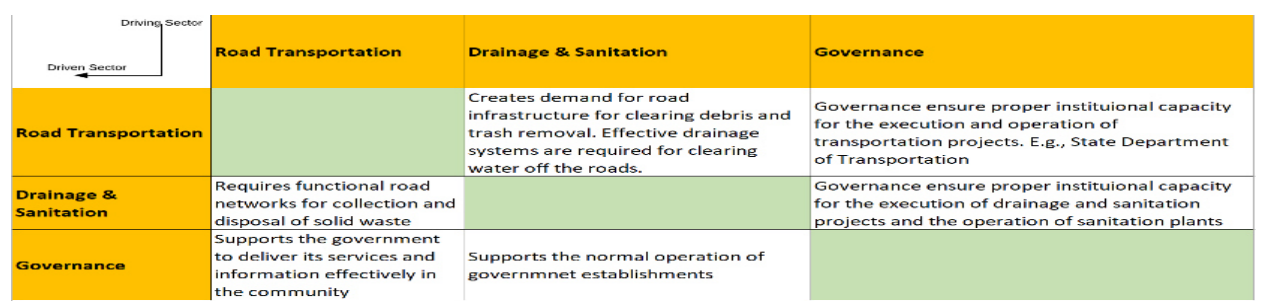

Figure 2 A Portion of the Level 1 Hypothesis DDSM

The Level 2 matrix of the DDSM (for technical managers) provides a detailed description of the interactions reported in the Level 1 matrix. For example, "Effective drainage systems are required to be in place to ensure that surface water such as rainwater is drained without disrupting the normal operations of road networks. Further, sanitation services require functional road networks for garbage trucks to collect waste and for treatment plants and landfill operations" is an interaction reported in hypothesis Level 2 matrix between Drainage \& Sanitation (Driving Sector) and Road Transportation (Driven Sector) that supports the Level 1 description shown in Figure 2.

\section{Hypothesis Testing}

\subsection{The Case Study DDSM}

$\mathrm{J} \& \mathrm{~K}$ was chosen as the case study to compare and analyze the community infrastructure system interactions in the hypothesis DDSM. Two disruptions to normal community functioning were used to identify recovery infrastructure sectors and their interactions, one natural disruption, and one man-made disruption. The natural disaster is the flooding in September 2014, which caused massive damage, killing more than 300 people and destroyed houses, educational institutes, crops, government establishments, businesses, etc. (Tabish and Nabil 2014). The man-made disruption was due to the 2019 political unrest in Jammu and Kashmir, after which the Indian government discontinued internet services in the state and shut down the traditional communication network. Economic losses of \$1 
billion were reported with significant disruptions caused in the education and public health sectors (Siddiqui 2019).

News and scholarly articles published between September 2014 and December 2019 that reported the impacts of disruptions on the community infrastructures were used to identify which infrastructures sectors were impacted and how. The reported case study infrastructure interactions were modeled as a DDSM. Although similar to hypothesis DDSM, the case study DDSM provides descriptions of the interacting cells based on actual interactions, as reported in case study literature. For example, "Effective sanitation infrastructure services are required to drain off the rainwater that may otherwise cause waterlogging on the roads in $\mathrm{J}_{\mathrm{K}} \mathrm{K} "$ is an interaction reported in the Level 1 case study matrix between Drainage \& Sanitation (Driving Sector) and Road Transportation (Driven Sector).

Figure 3 shows a snapshot of a portion of Level 1 of Final DDSM. The complete final DDSM is too large to show here but is available from the authors.

\begin{tabular}{|c|c|c|c|}
\hline 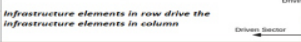 & Tourism & Communication/ICT & Governance \\
\hline Tourism & & $\begin{array}{l}\text { Information infrastructure is required } \\
\text { by the tourists to manage their travel } \\
\text { schedules and by the local tourism } \\
\text { industry to manage the visiting } \\
\text { tourists. }\end{array}$ & $\begin{array}{l}\text { Governance provides safety, } \\
\text { secuirty and estlablishes order } \\
\text { in the community. }\end{array}$ \\
\hline Communication/ICT & $\begin{array}{l}\text { Creates demand for information } \\
\text { infrastructure for supporting tourism } \\
\text { operations such as making travel } \\
\text { bookings etc. }\end{array}$ & & $\begin{array}{l}\text { Government regulates the flow } \\
\text { of information in the } \\
\text { community and also ensures } \\
\text { proper instituional capacity to } \\
\text { meet the demands. }\end{array}$ \\
\hline Governance & $\begin{array}{l}\text { Tourism creates a demand for } \\
\text { government to regulate order in the } \\
\text { community for safety and secuirity. } \\
\text { Tourism also creates revenue for the } \\
\text { government in the form of taxes for } \\
\text { the functioning of the government }\end{array}$ & & \\
\hline
\end{tabular}

Figure 3 A portion of Level 1 Final DDSM Model

\subsection{Comparison of the Hypothesis DDSM and Case Study DDSM}

The hypothesis DDSM was compared with the case study DDSM and the differences analyzed to assess the validity and usefulness of the DDSM and for model improvement. Both the number and location of interactions were examined. Ninety-three percent (180 of 193) of interactions in the hypothesis DDSM were reported in the case study DDSM. Twelve interactions were found missing in the case study DDSM that are reported in the hypothesis DDSM because of the case study's specific cultural and economic context. As such, interactions not reported in the case study are still assumed to hold in other communities with different contexts. One interaction was found missing in the hypothesis DDSM that was reported in the case study DDSM. The hypothesis DDSM was updated to include this interaction to create the final DDSM. Based on the case study tests, the final DDSM is judged to be useful for analyzing the resourcing of community recovery from disasters. 
Qureshi, Rameez; Ford, David N.; Wolf, Charles M.

\section{DDSM Model Analysis}

The final 16 by 16 DDSM was analyzed using Cambridge Advanced Modeler (CAM)@, a software tool used for modeling and analyzing flows and dependencies in complex systems (Wynn, 2010). Since CAM only identifies the cells of interaction and not the contents of the interacting cell, all three levels of Final DDSM, when modeled in CAM, yield the same DSM (in terms of if an interaction exists or not). The structure of the DSM was analyzed using Structural Profiling in CAM that uses centrality metrics from Social Network Analysis (SNA) to measure and analyze the structural properties of complex networks. The structural profiling analysis was used to characterize individual sectors (nodes) in the system in terms of their criticality for recovery. The following metrics from SNA were used in the analysis: Betweenness Centrality (measures which nodes are 'bridges' between nodes in a network by identifying all the shortest paths and counting how many times each node falls on the shortest path); In-Degree Centrality (implies the number of infrastructure services that drive/constrain an infrastructure sector); Out-Degree Centrality (means the number of infrastructures that are driven/constrained by another infrastructure sector).

According to Wynn (2010), higher centrality metrics values can reflect a sector's importance or criticality. For example, sectors with higher betweenness centrality influence the volume of the flows of people, materials, and information through a system and are therefore critical for an unconstrained recovery of the community. Also, the current work defines the terms - critical infrastructure and critical interaction. A critical infrastructure (including both physical and social infrastructures) is one that provides essential services for community operations and has a significant influence on the recovery of at least one other sector. A critical interaction between two infrastructures is a dependency that can constrain the capacity of the dependent (driven) infrastructure. Table 1 shows the centrality values of the community infrastructure sectors in the final DDSM, categorized as critical, semi-critical, and other based on their centrality values.

Table 1 Centrality Values of Community Infrastructure Sectors

\begin{tabular}{|c|l|c|c|c|}
\hline No & Sector & $\begin{array}{c}\text { Betweenness } \\
\text { Centrality }\end{array}$ & $\begin{array}{c}\text { In-Degree } \\
\text { Centrality }\end{array}$ & $\begin{array}{c}\text { Out-Degree } \\
\text { Centrality }\end{array}$ \\
\hline 1 & Commerce & 6.37 & 15 & 15 \\
\hline 2 & Workforce Population & 6.19 & 15 & 14 \\
\hline 3 & Road Transportation & 5.23 & 13 & 15 \\
\hline 4 & $\begin{array}{l}\text { Power-Gen \& } \\
\text { Distribution }\end{array}$ & 5.03 & 14 & 14 \\
\hline 5 & Governance & 2.7 & 10 & 15 \\
\hline 6 & Gasoline & 3.13 & 12 & 14 \\
\hline 7 & $\begin{array}{l}\text { Water Supply \& } \\
\text { Waste Treat. }\end{array}$ & 3.3 & 13 & 11 \\
\hline 8 & Public Health & 2.64 & 14 & 12 \\
\hline & & \multicolumn{3}{|c}{} \\
\hline 9 & Food & 1.95 & 13 & 11 \\
\hline
\end{tabular}




\begin{tabular}{|c|l|c|c|c|}
\hline No & Sector & $\begin{array}{c}\text { Betweenness } \\
\text { Centrality }\end{array}$ & $\begin{array}{c}\text { In-Degree } \\
\text { Centrality }\end{array}$ & $\begin{array}{c}\text { Out-Degree } \\
\text { Centrality }\end{array}$ \\
\hline 10 & Tourism & 1.73 & 12 & 12 \\
\hline 11 & Communication & 1.54 & 11 & 11 \\
\hline 12 & $\begin{array}{l}\text { Drainage and } \\
\text { Sanitation }\end{array}$ & 1.24 & 10 & 10 \\
\hline 13 & Education & 1.09 & 10 & 10 \\
\hline 14 & Other Transportation & 0.69 & 10 & 10 \\
\hline 15 & Residential Housing & 0.17 & 8 & 6 \\
\hline 16 & Resident Population & 5.01 & 13 & 13 \\
\hline
\end{tabular}

\subsection{Final DDSM Model Analysis Results}

\subsubsection{Critical Infrastructures for Recovery}

Sectors that provide services to all the other 15 infrastructures (Out-Degree Centrality $=15$ ) OR those that lie on the most number of shortest paths between sectors (Betweenness Centrality >5) were identified as the most critical infrastructure sectors for community recovery. Based on their centrality values, the five most essential (critical) infrastructure sectors for community recovery are - Commerce, Governance, Power Distribution and Generation, Road Transportation, and Workforce Population. These five most critical sectors provide the basic services required for the recovery of all other infrastructure sectors. These five critical sectors can be considered "foundation" infrastructures because they create and sustain the structures upon which other infrastructures and the recovery are built. Therefore, the primary focus of resourcing in the recovery period should be on the restoration of these five infrastructure services that are required for the recovery of other infrastructures. These results do not suggest that the other community sectors are not necessary, only that they are less critical to fast and efficient recovery than the sectors assessed to be most critical.

\subsubsection{Critical Infrastructure Interactions for Recovery}

Identifying and knowing the most critical community sectors for recovery is necessary but not sufficient for designing effective recovery strategies. Community leaders must also consider the interactions between sectors. The five most critical infrastructure sectors form a core set of causal feedback loops for recovery. Each of the five most critical sectors drives or constrains, and is driven or constrained by, the other four sectors, creating a very tightly coupled system. Therefore, constrained capacity in any one of these sectors constrains the others and thereby community recovery, and likewise, they support each other and promote recovery in each other. Note that this is irrespective of whether the limited capacity is due to the disaster, pre-existing conditions, or other causes. Therefore, for fast and effective recovery, community leaders must design and manage sector interactions as well as the restoration of individual sectors. For example, not all activities aid community recovery equally because not all infrastructure recovery actions help build capacity in other infrastructures. To speed community recovery infrastructures must recover in ways that 
build the capacity of their dependent infrastructures. Community recovery planners should target recovery efforts that restore and support dependent infrastructures.

In summary, five interacting community sectors identified as critical for recovery form core loops necessary for the recovery and operation of other sectors. A focus on sector interactions can help identify more and less effective recovery activities based on sector dependencies. Recovery designers and managers should assess the condition and capacity of all critical sectors after a disaster to identify the resource requirements for keeping the core recovery sectors from forming any bottlenecks in the recovery process. The DDSM can be a useful tool to identify the critical sectors and their interactions in the post-disaster period.

\section{Conclusions}

Infrastructure sector interdependencies can have significant impacts on community recovery from disasters. The current work identified critical infrastructure systems to understand how interactions among these sectors drive and constrain recovery. A threelevel Descriptive Design Structure Matrix (DDSM) for non-technical and technical managers was developed to define and identify the interactions between community sectors. The DDSM can be a useful tool to aid community leaders in understanding the impacts of resource allocation strategies. The DDSM provides simple, logical descriptions of community infrastructure interactions that can create recovery bottlenecks and a systematic and structured approach to resourcing recovery. The tool can also help identify pre-disaster vulnerabilities that may impact the recovery of infrastructure sectors in the post-disaster period, and therefore aid in developing policies for building self-sustaining and resilient communities. The insights drawn from the DSM model could constitute the core elements of a normative approach to community recovery for developing resilient recovery strategies. Improving the descriptions of community sectors that are critical to disaster recovery and their interactions can enhance the understanding of the drivers and constraints of recovery and thereby facilitate the development and implantation of effective, timely, and efficient community recovery.

References

Amin, M. 2002. "Toward Secure and Resilient Interdependent Infrastructures." Journal of Infrastructure Systems, 8(3), 67-75.

Browning, T. (2016). "Design Structure Matrix Extensions and Innovations: A Survey and New Opportunities." IEEE Transactions of Engineering Management, Vol. 63 (1) 27-52

Eppinger, S., and T. Browning. 2012. Design Structure Matrix Methods and Applications. The MIT Press, Cambridge, Massachusetts.

Ford, D., and B. Keith. 2016. "A Dynamic Feedback Theory of Community Recovery from Shock Events: Testing the role of social capital." System Dynamics Conference, Delft, The Netherlands.

GFDRR. 2015. Developing Disaster Recovery Frameworks - Sendai Conference version. GFDRR, World Bank Group, Washington D.C.

Ouyang, M. 2014. "Review on modeling and simulation of interdependent critical infrastructure systems." Reliability Engineering and System Safety, (121) 43-60.

Lindell, M. K., R. W. Perry, C. Prater, and W. C. Nicholson. 2006. Fundamentals of Emergency Management. FEMA Emergency Management Institute, Emmitsburg, Maryland. 
Olshansky, R. B., L. D. Hopkins, and L. A. \& Johnson. 2012. "Disaster and recovery: Processes compressed in time." Natural Hazards Review, 13(3) 173-178.

National Governors' Association (NGA). 1979. Comprehensive Emergency Management - A Governors' Guide. Centre for Policy Research, Washington, D.C.

Rand, K., and C. Fleming. 2019. "An Interdisciplinary review to develop guidelines for modeling population displacement as a function of infrastructure reconstruction decisions." Transportation Research Interdisciplinary Perspectives, 3(2019) 100072.

Rinaldi, S., J. Peerenboom, and T. Kelly. 2001. "Identifying, Understanding, and Analyzing Critical Infrastructure Interdependencies." IEEE Control Systems Magazine 21 11-25.

Siddiqui, Z. 2019. "Kashmir Shutdown Caused Losses of More Than \$1 Billion, Trade Body Says". Reuters, November 19.

Tabish, S., and S. Nabil. 2014. "Epic Tragedy: Jammu \& Kashmir Floods: A Clarion Call." Emergency Medicine, 5:1 (1000233).

Wynn, D., Wyatt, D., Nair, S., \& Clarkson, P. 2010. "An Introduction to the Cambridge Advanced Modeller." Proceedings of MMEP 2010, Cambridge, UK.

Corresponding Author: Rameez R. Qureshi; Graduate Student; Zachry Department of Civil and Environmental Engineering, Texas A\&M University, College Station, TX 77843 Email: rameez.rq@tamu.edu 